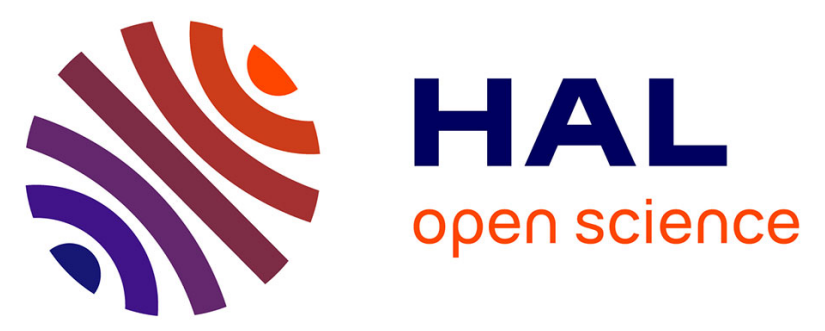

\title{
Management of product characteristics uncertainty based on Formal Logic and Characteristics Properties Model
}

Jean-Yves Dantan, Ahmed Jawad Qureshi, Jean-François Antoine, Boris Eisenbart, Lucienne Blessing

\section{To cite this version:}

Jean-Yves Dantan, Ahmed Jawad Qureshi, Jean-François Antoine, Boris Eisenbart, Lucienne Blessing. Management of product characteristics uncertainty based on Formal Logic and Characteristics Properties Model. CIRP Annals - Manufacturing Technology, 2013, 62 (1), pp.147-150. 10.1016/j.cirp.2013.03.077 . hal-02519006

\section{HAL Id: hal-02519006 \\ https://hal.science/hal-02519006}

Submitted on 25 Mar 2020

HAL is a multi-disciplinary open access archive for the deposit and dissemination of scientific research documents, whether they are published or not. The documents may come from teaching and research institutions in France or abroad, or from public or private research centers.
L'archive ouverte pluridisciplinaire HAL, est destinée au dépôt et à la diffusion de documents scientifiques de niveau recherche, publiés ou non, émanant des établissements d'enseignement et de recherche français ou étrangers, des laboratoires publics ou privés. 


\title{
Management of product characteristics uncertainty based on Formal Logic and Characteristics Properties Model
}

\author{
J.Y. Dantan (2)a, A.J. Qureshib, J.F. Antoinea, B. Eisenbartb, L. Blessingb \\ ${ }^{a}$ LCFC, Arts et Métiers ParisTech Metz, 4 Rue Augustin Fresnel, 57078 METZ CEDEX 3, France. \\ ${ }^{b}$ Research Unit in Engineering Science, University of Luxembourg, Luxembourg
}

Uncertainty in product characteristics is ubiquitous in any engineering system at all the stages of product life-cycle. Considering uncertainty from different sources during the product design phase is critical to its reliable performance. This paper presents a framework in tegrating the uncertainty propagation through different product characteristics and its effect on product properties. The framework consists of three main parts: a descriptive model based on formal logic and characteristics properties model; a mathematical implementation through set theory and probabilistic approach; and an algorithm for design space evaluation and tolerancing. The application of framework is demonstrated through an industrial case study.

\section{Introduction}

UNCERTAINTY is ubiquitous in any engineering system at all the stages of product development and throughout a product life cycle. Examples of uncertainty are manufacturing imprecisions, usage variations and manufactured geometric dimensions, which are all subjected to incomplete information. Such uncertainty has a significant impact on product performance.

For the embodiment design phase, the most commonly used methods to deal with uncertainty refer to Reliability BaseD Optimization (RBDO). RBDO methods are based on the probability distributions to describe variability of design variables and model parameters. They intend to achieve systems with an acceptable level of reliability (failure probabilities) and a satisfying level of performance. A solution is said to be reliable if the probability of satisfying each constraint is greater than a specified reliability level. RBDO methods consist of design optimization with a reliability assessment [1].

While RBDO methods concern the probability of constraints satisfaction, Robust Design Optimization (RDO) aims at minimizing the variations of the performance under uncertainty. There is still not a clear definition of robustness, most of the authors agree to define that a robust solution is a solution having minimum possible sensitivity to all sources of uncertainty. RDO methods intend to achieve systems with slight performance variations around their nominal values.

In the detail design phase, to ensure the desired behaviour and the functional requirements of the mechanism in spite of uncertainties (manufacturing imprecisions), the component features are assigned a tolerance zone within which the value of the feature i.e. situation and intrinsic lie. Therefore, tolerance design is a key element in industry for improving product quality and decreasing the manufacturing cost. In the last decade, a lot of methods have been developed to analyse tolerances.
All the major design processes address the uncertainty management through two major analysis and integration activities by using different tools. These activities are the robust design activity and the tolerance design activity. In order to put these into practice, different types of tools and methods specific to each of the above exist.

However, currently, no formal framework exists that allows the expression of the design problem at hand in a quantifiable manner while integrating the expressions of uncertainty.

Design is a decision based process in which a designer has to advance by making decisions about the product design while keeping into account the different constraints related to the product [2]. These constraints stem from the clients requirements that are translated into qualitative and quantitative forms via a variety of methodologies and processes such as axiomatic design [3], Function Behavior Structure [4], Key characteristics [5], House of quality, Pugh charts etc. The design process can be divided into stages, each of which may contain many sequential or concurrent activities appearing once or iteratively throughout the process. Due to the iterative nature of the design, these activities may reoccur throughout the the design process irrespective of the degree of maturity of the process. As the process evolves, depending on the degree of maturity, the process will proceed from qualitative to quantitative, from the concept to the concrete [6]. The activities may be classified into activities requiring quantitative or qualitative decision making according to type of information. The quantitative decisions are based on the constraints and requirements which can be quantified explicitly or implicitly i.e. can be measured quantitatively directly or indirectly.

This paper proposes a formal framework based on concepts of First Order Logic that is applicable to the aspects of design problem solving with quantitative decision making affected by uncertainty. As these aspects are more evident in the embodiment and detailed design stages of a design process, 
therefore this framework is applicable to these stages with the prerequisite of constraints being known.

The definition and the classification of uncertainty are discussed in the section 2. Based on this classification, a formal language to manage uncertainty is proposed in the section 3 , and it is applied to the reliability design and tolerance design. The application of framework is demonstrated through an industrial case study in the last section.

\section{Uncertainty}

One of the most controversial discussions in uncertainty analysis relates to the classification of uncertainty into several types and the possible sources from where it emanates. A classical classification is the separation of uncertainty into the two types: aleatory and epistemic. Aleatory uncertainty, also referred to as irreducible, objective or stochastic uncertainty, describes the intrinsic variability associated with a physical system or environment. According to the probability theory, aleatory uncertainty is modeled by random variables or stochastic processes. Epistemic uncertainty, on the other hand, is due to an incomplete knowledge about a physical system or environment. Study on epistemic uncertainty due to the lack of knowledge has received increasing attention in risk assessment, reliability analysis, decision-making, and design optimization. Epistemic uncertainty is sometimes referred to as state of knowledge uncertainty, subjective uncertainty, or reducible uncertainty, meaning that the uncertainty can be reduced through increased understanding (research), or increased availability of relevant data. Epistemic quantities are sometimes referred to as quantities which have a fixed value in an analysis, but we do not know that fixed value [7].

In the same way, [8] and [9] distinguish four main types of robust design. The type I robust design aims to identify design variables that satisfy the design requirements despite variation in noise factors (aleatory uncertainty). Type I robust design was proposed by Taguchi. Type II robust design aims to identify the design variables that satisfy the design requirements despite the variations (aleatory uncertainty) in the design variables themselves. Type III robust design identifies the adjustable ranges for design variables that satisfy the set of performance requirement targets and are insensitive to variability (epistemic uncertainty) within the system model. Type IV robust design identifies the adjustable ranges of design variables under potential uncertainty (aleatory and epistemic uncertainty) and uncertainty propagation in a design and analysis process chain; accounts for uncertainty in downstream activities and uncertainty propagation.

In the following, we focus on the types I, II and III of the robust design.

Example: the uncertainty of the relationship between the delivery $\mathrm{Q}[\mathrm{l} / \mathrm{min}]$, the displacement $\mathrm{V}[\mathrm{cm} 3 / \mathrm{rev}]$ and the drive speed $\mathrm{N}$ of a gear pump is illustrated:

$\mathrm{Q}=\mathrm{V} \cdot \mathrm{N} \cdot \eta_{\mathrm{V}} \cdot 10^{-5}$ with $\eta_{\mathrm{V}}$ : the volumetric efficiency

The model describing the relation between $\mathrm{N}$ and $\mathrm{Q}$ is not a simple mathematical function. It is a possibility domain due to:

- the uncertainty of the volumetric efficiency, the delivery model is affected by an epistemic uncertainty, which impacts the type III robust design.

- the uncertainty in V (displacement) due to aleatory factors such as manufacturing errors, tolerancing zones which impacts the type I robust design.

- the uncertainty in drive speed $\mathrm{N}$ due to aleatory factors which impacts the type II robust design

\section{Formal framework for uncertainty management}

A formal language is required that allows the mathematical and logical expression capable of describing the interactions between different parameters and constraints in the quantitative design problem clearly. The objective of quantitative design activities is to find the values of the key parameters in a design problem which satisfy the requirements. These key parameters may be involved in relations that dictate the fundamental properties related to product physics and performance. These relations are the design constraints. Therefore, a quantitative design problem can be modeled as a problem composed of a finite set of design variables, and a set of design constraints that restricts the values the variables can simultaneously take [10].

The proposed formal framework is based on the grammar of the First Order Logic (FOL) [11] and the framework of Characteristics Properties Model (CPM) [12], [13]:

- $\quad$ Characteristics (C) "describe the shape and the structure of a product (e.g. geometry, materials etc.) and can be directly established, assigned and modified by the designer. Characteristics may be quantitatively represented in form of dependent and independent variables. Characteristics represent the set of all design variables in the design problem.

- Properties (P) "describe the current behavior of a product (e.g. weight, manufacturability, function, cost, user friendliness etc.) and cannot be directly established by the designer; they can only be indirectly influenced by changing the depending characteristics" [14]. They are the indicator of the performance of the product, resulting from a given set of characteristics. The set of properties is defined as:

- $\quad$ Required Properties (RP) The required properties are the properties that are to be achieved in order for the design process to succeed.

- Design Space (D) The characteristics are the variables to which values must be assigned. These assignments are taken from the design space. This denotes the ndimensional space in which the solution is to be searched. D consists of the sets of domains of the characteristics which limit the values that can be assigned to them.

- Relations (R) relate the characteristics to the properties through the laws of physical behavior and tangible/intangible principles. Relations may be deduced from physical objects (models, mockups, and prototypes) or they may be made in a non-physical model (mathematical, numerical, computer-based, graphical, etc.).

- External Conditions (EC) Essentially, uncertainty in CPM may originate from external conditions EC e.g. coming from the environment or associated technical systems, which may impact on the relation between characteristics and properties. External conditions are the properties of a system $\mathrm{X}$ that have an impact on the relation linking the characteristics to the properties.

- Modeling Conditions (MC) are the set of assumptions, hypothesis, boundary conditions or simplifications used while developing the model that have to be taken into account for defining the relations between the characteristics and the properties.

The proposed framework defines the unified high level expressions of 3 different levels of increasing robustness. Being based on logic and CPM, the framework can be applied to most of constraint satisfaction problems encountered in the embodiment and detailed design phase of mechanical systems design. Two areas of possible applications are discussed in this section. 
The first application is an exploration of the design space during the embodiment design to find solutions with an acceptable level of reliability and a satisfying level of performance. The reliability design aims to assure that the product performance remains acceptable under the influence of uncertainty. To illustrate the FOL formulation, we focus on Type II robust design. Type II robust design aims to identify the characteristics that result into required performance despite the variations in the characteristics themselves. This condition can be satisfied by evaluating for a set of values of characteristics instead of a single value. This is achieved through the "universal" quantifier. The solution for the type II robust design is expressed as:

$$
\exists s_{i} \mid s_{i} \subseteq D
$$$$
S_{I I}=\left\{\bigcup_{\text {sieS }}, S_{i} \vDash \forall C \forall E C R(C, P, R P, E C)\right\}
$$

$S_{I I}$ denotes the Type II robust design solution. The above expression assures that the valid solution accounts for the variation in the characteristics as well as the full range of the external conditions and that any variation in both would not compromise the properties of the product below the threshold of the required properties. The above expression not only expresses the Type II robust design but also describes the essential conditions for the Set Based Design as described by [15]. The solutions validating the above expressions can therefore be termed as set-based robust solutions as in addition to the solution being in terms of sets; it also integrates the uncertainty factors as well. [10] proposes an algorithm using Quantified Constraint Satisfaction Problem (QCSP) transformation to perform the reliable solution space search.

The second application is the expression of functional defect probability for a tolerance design problem. [16] proposes a quantified formulation of the tolerance analysis problem which is generalized in this proposed framework: In this case, the part deviations are aleatory uncertainties modeled by external conditions $\left(\boldsymbol{E} \boldsymbol{C}_{\boldsymbol{m}}\right)$ (they are the properties of an external system X: the manufacturing imprecision) and the gaps between parts $\left(\boldsymbol{P}_{\boldsymbol{g}}\right)$ are epistemic uncertainty (due to the complexity of system behavior with gaps, epistemic uncertainty exists in the behavior model; for the tolerance analysis, we don't know the gap configuration; moreover, the worst gap configurations of the over-constrained system depend on the component deviations). The relations which model the mechanism behavior and functionalities could be divided in three types: $\boldsymbol{R}_{\boldsymbol{c}}$ (compatibility relations which represent the topological constraints), $\boldsymbol{R}_{i}$ (interface relations which represent the geometrical behavior of the contact between parts), and $\boldsymbol{R}_{\boldsymbol{f}}$ (functional relations which represents the required properties). The set of properties $\boldsymbol{P}$ contains two types: $\boldsymbol{P}_{\boldsymbol{f}}$ (properties related to functional requirements) and $\boldsymbol{P}_{\boldsymbol{g}}$. We can formalize the expression of the functional defect probability: "the probability of the non-respect of the functional requirement for all admissible gap configurations which is limited by the compatibility relations and the interface relations".

$$
\begin{aligned}
& \text { Probadefect }_{\text {Proba }}\left(P _ { f } \notin \left\{\bigcup_{s i \in S},\right.\right. \\
& \left.\left.\mathrm{S}_{\mathrm{i}} \vDash \forall P_{g} \exists P_{f} R_{c}\left(E C_{m}, P, C\right) \wedge R_{i}\left(E C_{m}, P, C\right) \wedge R_{f}\left(E C_{m}, P, C\right)\right\}\right)
\end{aligned}
$$

\section{Illustration}

The proposed framework can be used to model a variety of design problems. In this section, an example of sizing an external gear pump is presented. The objective of the example is to search the design space for a reliable solution for selecting a pump according to the required properties of delivery rate and pressure via scaling a standard platform design. The reliable solution should perform according to the required properties in presence of different sources of uncertainty. Figure 1 shows the CPM diagram of an external gear pump for hydraulics application.

The example is based on the experimental data for the pump provided by the manufacturer in the form of pump curves with the variables $V$ (cm $3 / \mathrm{rev}$-Displacement), $Q$ (l/min-Delivery), $\Delta p$ (bar-Pressure), $M$ (Nm-Drive torque), $n$ ( $\mathrm{rev} / \mathrm{min}$ - Drive speed) and $p$ (kW-Drive power). The plots take into account the volumetric efficiency $\eta_{v}$ and hydraulic-mechanical efficiency $\eta_{\mathrm{hm}}$.

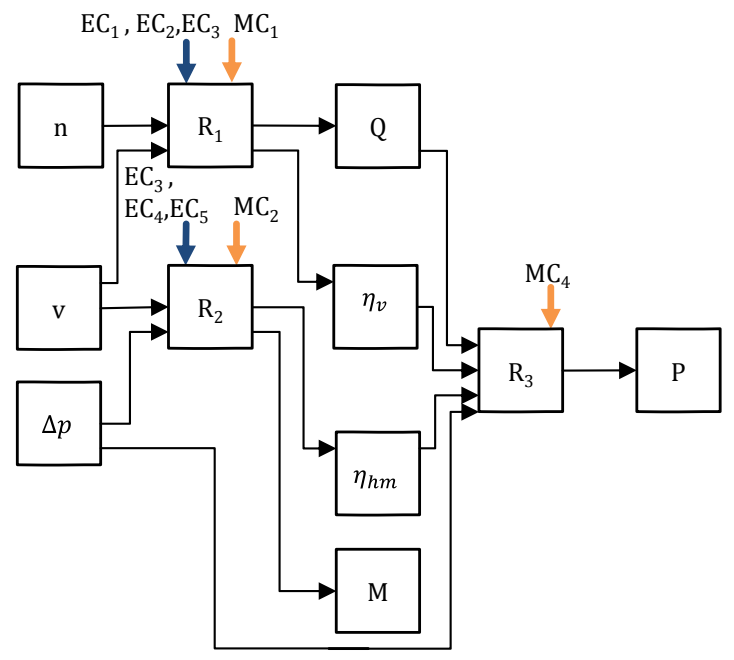

Figure 1. CPM diagram of an external gear pump

Three theoretical relations $\mathrm{R}_{1}, \mathrm{R}_{2}$, and $\mathrm{R}_{3}$ between $V, Q, \Delta p, M$, and $n$ as given by the manufacturer are given respectively as:

$$
\begin{aligned}
& p=\frac{\pi \cdot M \cdot \eta_{h m}}{5 \cdot V} \\
& P=\frac{\Delta p \cdot Q}{6 \cdot \eta_{t}}
\end{aligned}
$$$$
Q=V \cdot n \cdot \eta_{v} \cdot 10^{-3}
$$

Table 1. External Conditions and Modeling Conditions

\begin{tabular}{|l|l|l|}
\hline $\mathrm{EC} / \mathrm{MC}$ & Description & Range \\
\hline $\mathrm{EC}_{1}$ & Fluctuation in drive speed & {$[-13,13] \mathrm{RPM}$} \\
\hline $\mathrm{EC}_{2}$ & Displacement variation & {$[-0.5,0.5] \mathrm{Cm}^{3}$} \\
\hline $\mathrm{EC}_{3}$ & Leakage from Joints & Experimental \\
\hline $\mathrm{EC}_{4}$ & Power transmission loss & Experimental \\
\hline $\mathrm{EC}_{5}$ & Fluctuation in the back pressure & {$[-1,1]$ bar } \\
\hline $\mathrm{MC}_{1}$ & Meta Modeling error of $\eta_{v}$ & $0.1 \%$ \\
\hline $\mathrm{MC}_{2}$ & Meta Modeling error of $\eta_{\mathrm{hm}}$ & $2.33 \%$ \\
\hline $\mathrm{MC}_{3}$ & Simplification of overall efficiency $\left(\eta_{t}\right)$ & $\eta_{v} . \eta_{\mathrm{hm}}$ \\
\hline
\end{tabular}

In the given example of the gear pump, the revolutions per minute $n$, the pressure difference $\Delta p$, and the displacement $V$ of the pump can be directly influenced and thus represent characteristics $\left(\mathrm{C}_{1-3}\right)$ of the pump. The properties are dependent upon the characteristics, external conditions and modeling conditions. The properties considered in this example are the delivery $Q$, torque $M$, volumetric efficiency $\eta_{v}$, hydraulicmechanical efficiency $\eta_{h m}$, and required power $P$. It would be interesting to mention here that $P$ is a property that it is dependent on property, i.e., $P$ is a property of properties. The example uses 5 different external conditions to model uncertainty from external factors. Three Modeling conditions are also used to model the uncertainty due to modeling (Table 1 ). The required properties are a minimum delivery of $23 \mathrm{l} / \mathrm{min}$ and a maximum required power of $5 \mathrm{~kW}$. The characteristics to be decided are the values of $V[8,16] \mathrm{cm}^{3}$, and the drive speed $n[1500,3000] \mathrm{rpm}$. 


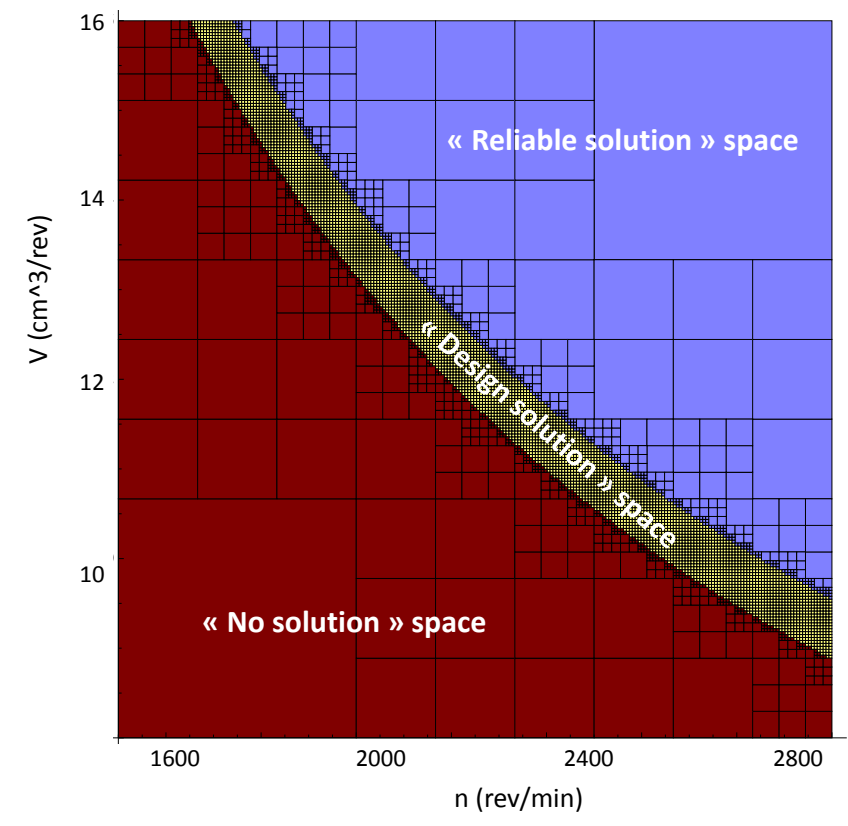

Figure 2. Type II robust design space

Using QCSP [10], it is possible to determine the Design solution space [13], and the "reliable solution" space for each type of robust design. For the example, Figure 2 shows the Type II robust design space: Each box is the permissible bounds related to the variation in the characteristics. The total number of "reliable solutions" boxes found in this example for the given design space D was 3092. The total number of solutions found in type III design decrease to 2256. This is due to the consideration of the modeling uncertainty related to the Meta models of the volumetric and hydraulic-mechanical efficiency.

\section{Conclusion}

In [13] the CPM/PDD (Property Driven Design) approach was used to explain the mathematical model of a mechanical design problem in redesign. It was shown, how the CPM may be modelled using formal language and mathematical constructs, in order to facilitate design automation in redesign. In this paper, the developed model was extended to account for the managing uncertainty in the design phase.

Essentially, uncertainty in CPM may originate from external conditions $\left(\mathrm{EC}_{\mathrm{j}}\right)$ e.g. coming from the environment or associated technical systems, which may impact on the relation between characteristics and properties.

The model presented in this paper proposes an approach for the simultaneous parameter and robust design activities in the quantitative design problems frequently encountered in redesign, and adaptive design problems. The model has the capacity to express the relations between the design variables and the uncertainty variables required to describe different types of robust design (Type I, Type II, Type III). Using the developed notion of quantifier by [16], this work provides a generalization and harmonization of the quantifier notion in a more structured and syntactic paradigm of formal logic for expressing quantitative design problems e.g. robust parametric design \& tolerance design.

The developed formalization expresses the search for a robust solution by using different quantified expressions that test the design space for the existence of a possible solution followed by evaluation of solution for Type I and Type II, Type III robustness. This integration renders the solution inherently robust and insensitive to the changes within the decided ranges. This formalization also makes it possible to carry out the robust design in a set based design process by manipulating and evaluating the sets of variables instead of points in design space which is the main premise of the set based design.

Another capability shown was the consideration of different modeling conditions that impact the individual relations and affect the depending properties. Their consideration is of importance as overlooking these may result into selection of solutions that while apparently fulfilling all criteria may fail due to these uncertainties.

Contrary to the objective of conventional robust design techniques which aim to evaluate a given optimized solution to sensitivity towards variation, the approach presented in this paper provides a set of solutions which incorporate the range of variations in the design space and variations in the variation space to provide a guaranteed set of solution. The designer can consequently choose between these solutions with optimization techniques to choose the solution that suits the requirements the most.

\section{Acknowledgements}

The authors acknowledge the support of ANR "AHTOLA" project (ANR-11- MONU-013) and the support of "TADMA" project of Luxembourg University.

\section{References}

[1] Roy R, Hinduja S, Teti R, 2008, Recent Advances in Engineering Design Optimisation: Challenges and Future Trends, CIRP Annals 57/2:697-715.

[2] Mistree F, Smith W, Bras B, Allen JK, Muster D, 1990, Decision-based design: contemporary paradigm for ship design, Transactions, Society of Naval Architects and Marine Engineers, Vol. 98, pp. 565-597.

[3] Suh N, 2001, Axiomatic Design- Advances and Applications, Oxford University Press.

[4] Gero J, 1990, Design prototypes: A knowledge representation scheme for design, AI Magazine, 11, 26-36.

[5] Thornton AC, 2003, Variation Risk Management, Focusing Quality Improvements in Product Developmment and Production, Wiley, UK.

[6] Pahl G, Beitz W, 2007, Engineering design: a systematic approach, Springer.

[7] Oberkampf WL, Deland SM, Rutherford BM, Diegert KV, Alvin KF, 2002, Error and Uncertainty in Modeling and Simulation. Reliability Engineering and Safety Systems, 75/3:333-357.

[8] Choi H-J, 2005, A robust design method for model and propagated uncertainty, $\mathrm{PhD}$ Thesis, Georgia Institute of Technology.

[9] Allen JK, Seepersad C, Choi, H, Mistree F, 2006, Robust Design for Multiscale and Multidisciplinary Applications, Journal of Mechanical Design, 128/4:832

[10] Qureshi AJ, Dantan JY, Bruyere J, Bigot R, 2010, Set Based Robust Design of Systems - Application to Flange Coupling, Proceedings of the CIRP Design Conference.

[11] Peters S, Westerstahl D, 2006, Quantifiers in Language and Logic, Oxford University Press.

[12] Weber C, 2007, Looking at "DFX" and "Product Maturity" from the Perspective of a New Aproach to Modelling Product and Product Development Processes, Proceedings of the CIRP Design Conference.

[13] Qureshi AJ, Eisenbart B, Dantan J-Y, Blessing L, 2013, Design automation with the characteristics properties model and the property driven design for redesign, Proceedings of $23^{\text {rd }}$ CIRP Design Conference.

[14] Köhler C, Jan C, Weber C, 2008, A Matrix Representation of the CPM/PDD Approach as a Means for Change Impact Analysis. Proceedings of International Design Conference.

[15] Sobek D K, Ward A C,Liker J K, 1999, Toyota's Principles of Set-Based Concurrent Engineering," Sloan Management Review, 40/2:67-83.

[16] Dantan J-Y, Gayton N, Qureshi A J, Lemaire M, Etienne A, 2012, Tolerance analysis approach based on the classification of uncertainty (aleatory / epistemic), Proceedings of 12th CIRP Conference on Computer Aided Tolerancing. 